\title{
Mapping Single Palm-Trees Species in Forest Environments with a Deep Convolutional Neural Network
}

\author{
Luciene Sales Dagher Arce ${ }^{1}$, Mauro dos Santos de Arruda $^{1}$, Danielle Elis Garcia Furuya ${ }^{2}$, Lucas Prado Osco ${ }^{3, \bigotimes}$, Ana Paula \\ Marques Ramos ${ }^{2}$, Camila Aoki ${ }^{1}$, Arnildo Pott ${ }^{1}$, Sarah Fatholahi ${ }^{4}$, Jonathan $\mathrm{Li}^{4}$, Wesley Nunes Gonçalvez ${ }^{1}$, and José Marcato \\ Junior ${ }^{1}$ \\ ${ }^{1}$ Faculty of Engineering, Architecture, and Urbanism and Geography, Federal University of Mato Grosso do Sul (UFMS), Avenida Costa e Silva, 79070-900, Campo Grande, \\ Mato Grosso do Sul, Brazil \\ ${ }^{2}$ Post-Graduate Program in Environment and Regional Development, University of Western São Paulo (UNOESTE), Rodovia Raposo Tavares, km 572-Limoeiro, 19067-175, \\ Presidente Prudente, São Paulo, Brazil \\ ${ }^{3}$ Faculty of Engineering and Architecture and Urbanism, University of Western São Paulo (UNOESTE), Rodovia Raposo Tavares, km 572-Limoeiro, 19067-175, Presidente \\ Prudente, São Paulo, Brazil \\ ${ }^{4}$ Department of Geography and Environmental Management, University of Waterloo (UW), Waterloo, ON N2L 3G1, Canada
}

\begin{abstract}
Accurately mapping individual tree species in densely forested environments is crucial to forest inventory. When considering only RGB images, this is a challenging task for many automatic photogrammetry processes. The main reason for that is the spectral similarity between species in RGB scenes, which can be a hindrance for most automatic methods. State-of-the-art deep learning methods could be capable of identifying tree species with an attractive cost, accuracy, and computational load in RGB images. This paper presents a deep learning-based approach to detect an important multi-use species of palm trees (Mauritia flexuosa; i.e., Buriti) on aerial RGB imagery. In South-America, this palm tree is essential for many indigenous and local communities because of its characteristics. The species is also a valuable indicator of water resources, which comes as a benefit for mapping its location. The method is based on a Convolutional Neural Network (CNN) to identify and geolocate singular tree species in a high-complexity forest environment, and considers the likelihood of every pixel in the image to be recognized as a possible tree by implementing a confidence map feature extraction. This study compares the performance of the proposed method against state-of-the-art object detection networks. For this, images from a dataset composed of 1,394 airborne scenes, where 5,334 palm-trees were manually labeled, were used. The results returned a mean absolute error (MAE) of 0.75 trees and an F1-measure of $86.9 \%$. These results are better than both Faster R-CNN and RetinaNet considering equal experiment conditions. The proposed network provided fast solutions to detect the palm trees, with a delivered image detection of 0.073 seconds and a standard deviation of 0.002 using the GPU. In conclusion, the method presented is efficient to deal with a high-density forest scenario and can accurately map the location of single species like the $M$ flexuosa palm tree and may be useful for future frameworks.
\end{abstract}

Convolutional Neural Network | Deep Learning | Environmental Monitoring Correspondence: lucasosco@unoeste.br

\section{Introduction}

The unplanned development and land occupation in both urban and rural areas are the main reasons behind deforestation, contributing to environmental degradation in riparian zones and modifying the natural landscape. Multidisciplinary re- search is necessary to ascertain the population of vegetative species, identifying their locations and distribution patterns. Such information is essential for the management and conservation of vulnerable ecosystems, and mapping these environments may help governmental entities to control or mitigate environmental damage properly. In the last decade, remote sensing data have been widely applied for monitoring vegetation health (1), biomass (2), forest management (3), biodiversity (4), among others (5-10). Thus, remote sensing approaches have been used to investigate areas with difficult terrain access, demonstrating great potential for the classification and detection of forest vegetation.

Remote sensing platforms can be embedded with different sensors such as RGB (Red-Green-Blue), multispectral and hyperspectral, LiDAR (Light Detection and Ranging), and others (11). The identification of arboreous vegetation with remote sensing data depends on the spatial and spectral resolutions (12). LiDAR sensors can produce accurate data on the height of the trees, which is an excellent variable to be adopted by automatic extraction methods in forest environments $(13,14)$. Multispectral and hyperspectral sensors have the advantage of recording the spectral divergence of the vegetation, which is important for enhancing differences between species configurations, health status, etc. $(10,15,16)$. Still, in recent years, high spatial resolution images acquired by RGB sensors have been used in many studies for vegetation identification $(9,17-20)$. These sensors have a relatively low cost in comparison with others but offer limited information regarding the spectral range.

For single-tree species mapping, the literature already investigated different methods by evaluating multispectral and hyperspectral data (21-26), airborne LiDAR point clouds (27), and multi-sensory data fusion (28-30). (31) were able to classify tree species in a temperate forest using satellite multispectral imagery. (32) evaluated UAV (Unmanned Aerial Vehicle)-based multispectral image to map deciduous vegetation. (33) used hyperspectral data to detect boreal tree species at pixel-level, achieving high accuracy for forest mapping. Most of these studies were conducted with hyperspectral sensors and LiDAR sensors. However, both hyperspectral and 
LiDAR data cost and process demand are non-attractive for rapid decision models. This is different from RGB sensors, which have a lower cost and are highly available. (34) demonstrated that the majority of recent applications are implementing RGB imagery data in the vegetation detection scenario.

The visual inspection of remote sensing imagery is a timeconsuming, labor-intensive, and biased task. Therefore, various studies have developed multiple methods regarding the automated extraction of the vegetation features $(10,15$, 22). Accurately mapping individual tree species in densely forested environments is still a challenging task, even for more robust methods. The increase in quality and quantity in remote sensing data, alongside the rapid improvement of technological resources, allowed for the development of intelligent methods in the computational vision community. By combining remote sensing data with artificial intelligence techniques, it is possible to properly map tree species and improve accuracy in applications regarding vegetation monitoring. In recent years, multiple frameworks have been implemented to assess the performance of such algorithms to accomplish this task $(2,5,15,35-37)$.

During the past years, the detection and extraction of trees in high-resolution imagery were performed with more traditional machine learning algorithms, like support vector machine (SVM), random forest (RF), artificial neural networks (ANN), and others (38-41). They returned interesting outcomes in plenty of studies regarding vegetation analysis (10, 42-46). However, these learners (known as shallow learners) are limited due to data complexity and may return lower accuracy in comparison with deep learning methods. When considering adverse conditions in a given forest dataset, deeper methods are required.

Identifying individual species in a scene can be a challenging task since (23). However, state-of-the-art deep learningbased methods should be capable of identifying single treespecies with an attractive accuracy and computational load even in RGB images. Recently, deep learning-based methods have been implemented in multiple remote sensing, specifically for image segmentation, classification, and object detection approaches (37, 47-49). Deep learning techniques are among the most recently adopted approaches to process remote sensing data (50-52). In a general sense, deep learning can return better performance than shallow learners, especially in the presence of large quantities of data or if the input data is highly complex $(53,54)$.

In heavy-dense forested environments, the identification of single-tree species can become a challenge even for robust methods like current state-of-the-art deep networks. This motivated several studies to investigate the performance of deep learning methods to evaluate their performance on this task. A recently published research tested the performance of object detection using deep networks like YOLOv3 (55), RetinaNet (56), and Faster-RCNN (57) to detect tree canopy in RGB imagery covering an urban area (9). Another study modified the VGG16 (58) to monitor the health conditions of vegetation (59). A combination of LiDAR and RGB images was also used with the RetinaNet to identify tree-crowns in UAV images (19). The DenseNet (60) was also implemented multispectral data to classify tree species.

The spatial and spectral divergences between the tree and non-tree are essential for automatic methods $(15,16)$. In highly-dense scenarios like heavily forested areas, the individual detection of trees could be difficult. RGB sensors are not capable of providing the same amount of spectral data as multispectral or hyperspectral sensors, which offers a potential hindrance for automatic extraction methods. Nonetheless, state-of-the-art deep learning methods based on confidence maps, instead of object detection approaches, could be capable of identifying single tree-species in highly dense areas using RGB images. Methods that could accurately identify a species, among others, may help optimize several applications in environmental planning and forest management. In the presented context, this paper presents a deep learning approach to detect individual fruit species of palm-trees (Mauritia flexuosa; L.f. Buriti) with only aerial RGB orthoimages. As contribution of this approach, a framework to identify and geolocate single species in a high-complex forested environment is demonstrated. The study compares the performance of the proposed method with other state-ofthe-art object detection deep neural networks to test its robustness. The palm tree M. flexuosa is a valuable source of food, remedy, fiber, and light wood for both indigenous communities and local populations (61-63). It is also considered a native species of the Brazilian flora with both current and potential high economic values $(62,64)$. Besides, this species has its ecological importance, constituting a food source, nest site, and habitat to a wide variety of species and provides multiple ecosystem services $(63,65,66)$, which highlights the need to accurately map this species.

\section{Materials and Method}

The method proposed in this paper is composed of three main phases (see Figure 1): (1) The dataset was composed of aerial RGB orthoimages obtained from a riparian zone of a wellknown populated region of $M$. flexuosa palm-trees. With specialist assistance, the palm-trees in the RGB images were visually identified and labeled in a Geographical Information System (GIS). The image and labeled data were split into groups of training, validation, and testing subsets; (2) The object detection approach was applied in a computational environment; (3) The performance of the proposed method was compared with other networks.

Study Area and Mapped Species. The riparian zone of the upper-stream of the Imbiruçu brook, located near the city of Campo Grande, in the state of Mato Grosso do Sul, Brazil was selected for the study (Figure 2). This stream, formed by a dendritic drainage system, is inserted in the hydrographical basin of the Paraguay River and covered by the Cerrado (Brazilian Savanna) biome. This area is composed of a highly complex forest patch containing a widespread of palm-tree species Mauritia flexuosa (popular name Buriti). The Arecaceae is a dioecious (67) long-living species and grows nat- 

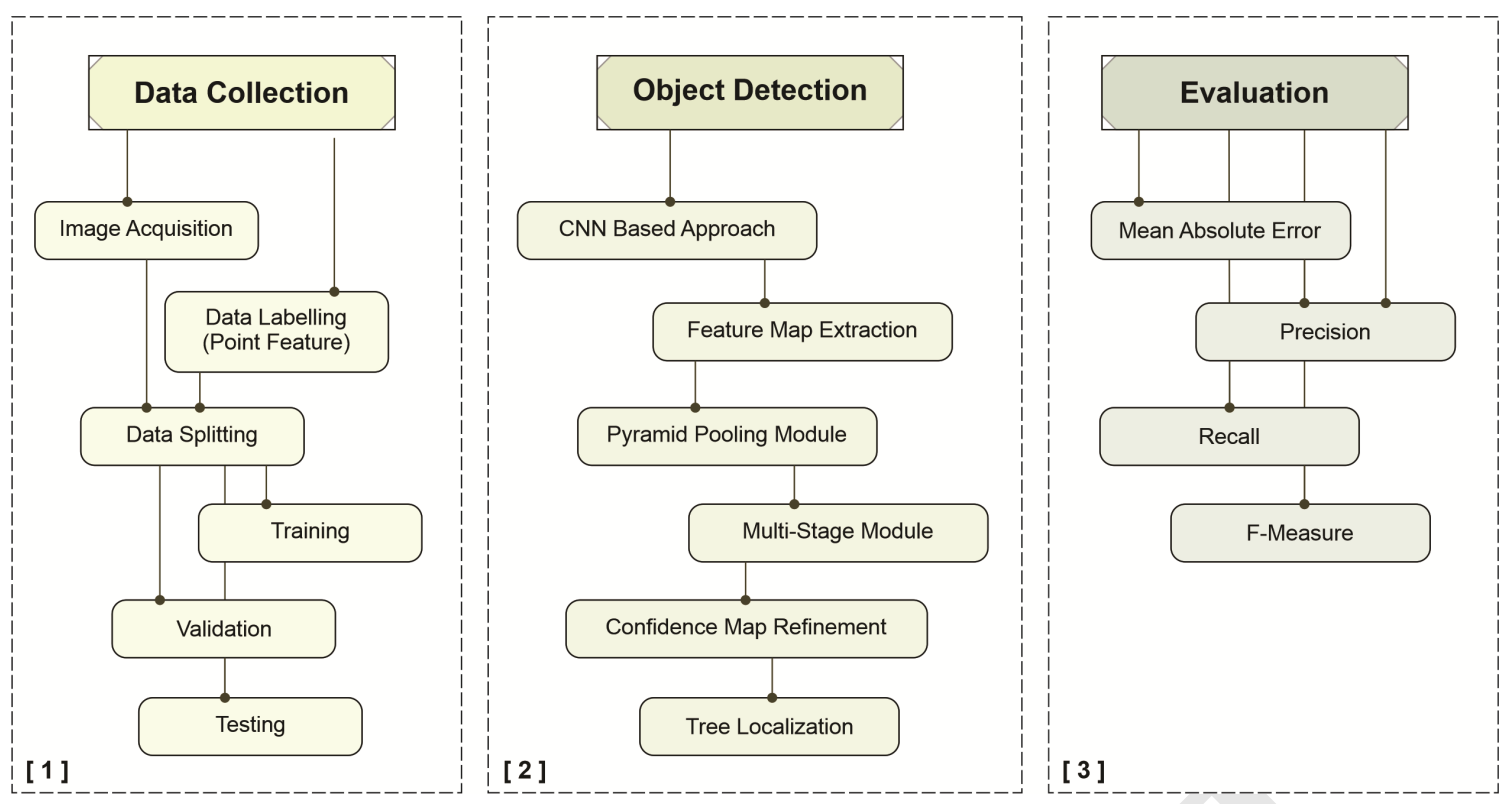

Fig. 1. Summarized phases of the proposed approach.

urally in flooded areas, providing water balance for rivers and other water bodies. In highly dense, monodominant stands in flooded areas, mature M. flexuosa palm trees reach $20 \mathrm{~m}$ high (67). The evaluated site in our experiment, in specific, is one of the well-known locations where a large number of samples of this species is sufficient to train a deep neural network.

The aerial RGB orthoimages were provided by the city hall of Campo Grande, State of Mato Grosso do Sul, Brazil. The ground sample distance (GSD) of the orthoimages is $10 \mathrm{~cm}$. A total of 43 orthoimages with dimensions 5,619 x 5,946 pixels were used in the study. This aerial image dataset was composed of 1,394 scenes, where 5,334 palm-trees were manually labeled and used as ground-truth (Figure 3).

Proposed Method. This study proposes a CNN method that uses the RGB image as an input and, throughout a confidence map refinement, returns a prediction map with tree locations (Figure 4). The objects' position is calculated after a 2D confidence map estimation, based on previous works (68). The first step of the architecture extracts the feature map. In a sequential step, the feature map goes through the Pyramid Pooling Module (PPM) (69). The last step of the architecture produces a confidence map in a Multi-Stage Module (MSM) that enhances the position of the tree by adjusting the prediction to its center.

Feature Map Extraction and PPM. For the feature map extraction (Figure 4(b)), the proposed CNN is based on the VGG-19 (58). Here, the network is composed of 8 convolutional layers with 64, 128, and 256 filters with a 3 x 3 size window, with Rectified Linear Units (ReLU) functions in all layers. The spatial volume size was reduced in half after the second and fourth layers by a max-pooling layer ( $2 \times 2$ window). The PPM (69) was used (Figure 4(c)) to extract global and local information, which helps the $\mathrm{CNN}$ to be less variant to tree scale differences. The extracted features are upsam- pled to size equivalent to the input feature map and concatenated with it to create an enhanced version of the image.

Tree Localization. The MSM step (Figure 4(d)) estimates the confidence map from the feature map extracted in the previous module. The MSM is composed of T refinement stages, where the first T stage contains 3 layers of 128 filters with $3 \times 3$ size, 1 layer with 512 filters of $1 \times 1$ size, and one final layer with 1 filter that generates the confidence map C1 from the first stage. The position of the trees predicted in the first stage is refined in the $\mathrm{T}-1$ stage phase. In each stage $\mathrm{T}$ $\mathrm{E}[2,3, \ldots ., \mathrm{T}]$, the prediction $(\mathrm{C})$ is returned from a previous stage ( $T$ - 1$)$ and the feature map from the PPM module is concatenated. The final layer in this step has a sigmoid activation function since the method considers the probability of occurrence of a tree to exist or not $[0,1]$. The concatenation process allows for both global and local context information to be incorporated in it. At the end of each stage, a loss function (1) is adopted to avoid the vanishing gradient problem. The general loss function is calculated by the following equation (2).

$$
f_{t}=\sum_{p}\left\|\hat{C}_{t}(p)-C_{t}(p)\right\|_{2^{\prime}}^{2}
$$

where $\mathrm{Ct}(\mathrm{p})$ is the ground-truth confidence map of location (p) in stage $(\mathrm{T})$.

$$
f=\sum_{t=1}^{T} f_{t}
$$

The confidence map is generated by a 2D Gaussian kernel at the center of the labeled tree. A standard deviation $\sigma$ t controls the spread of the peak for each Gaussian kernel (Figure $5)$. Different values of $\sigma \mathrm{t}$ were used to refine the predictions. The value of $\sigma 1$ in the MSM is set to maximum ( $\sigma \max )$ while the $\sigma \mathrm{t}$ in the final stage is set to minimum ( $\sigma \mathrm{min})$. In the 

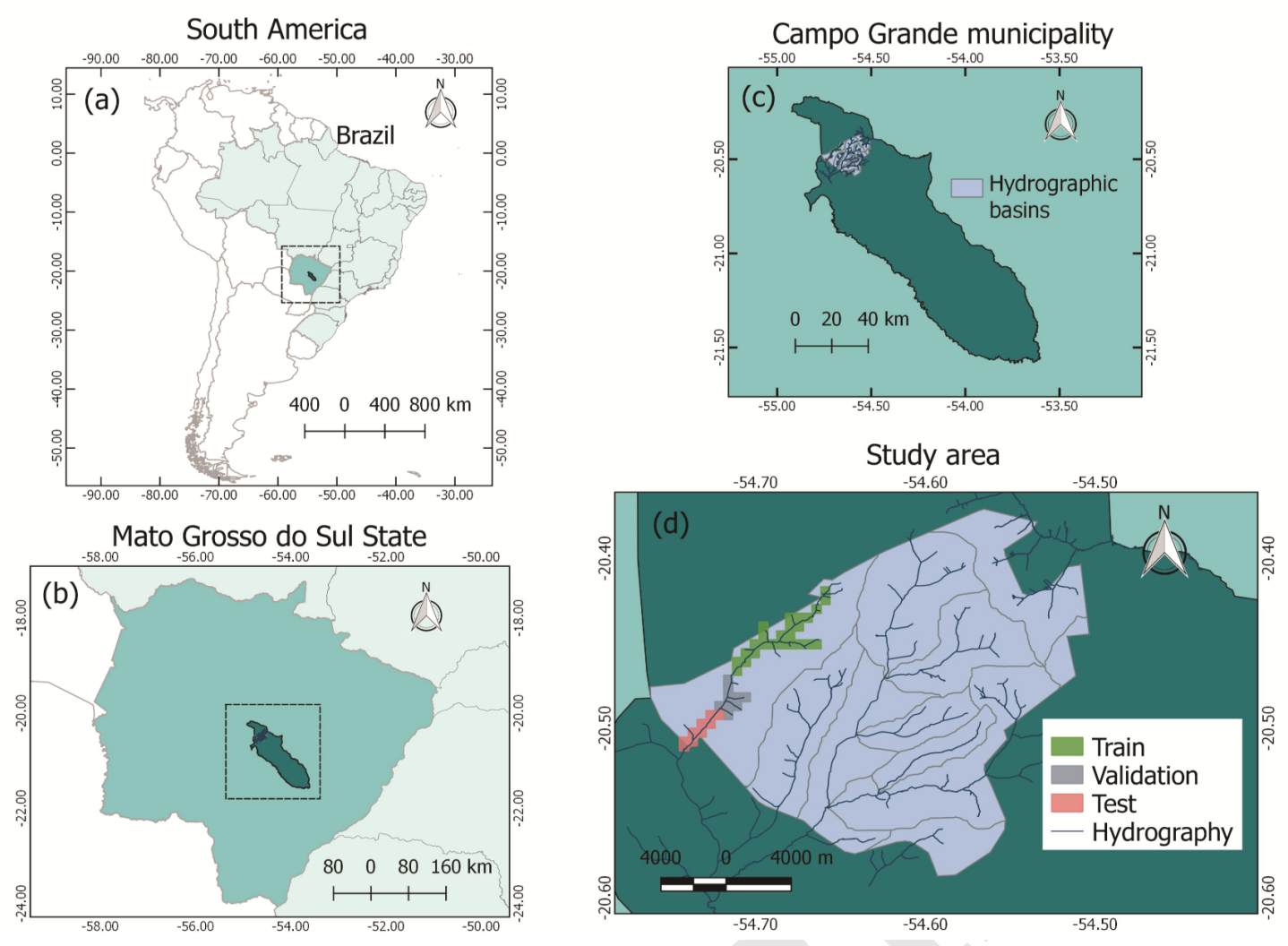

Fig. 2. Location map of the study area in (a) South America and Brazil, (b) Mato Grosso do Sul, (c) Campo Grande, and (d) Study area.

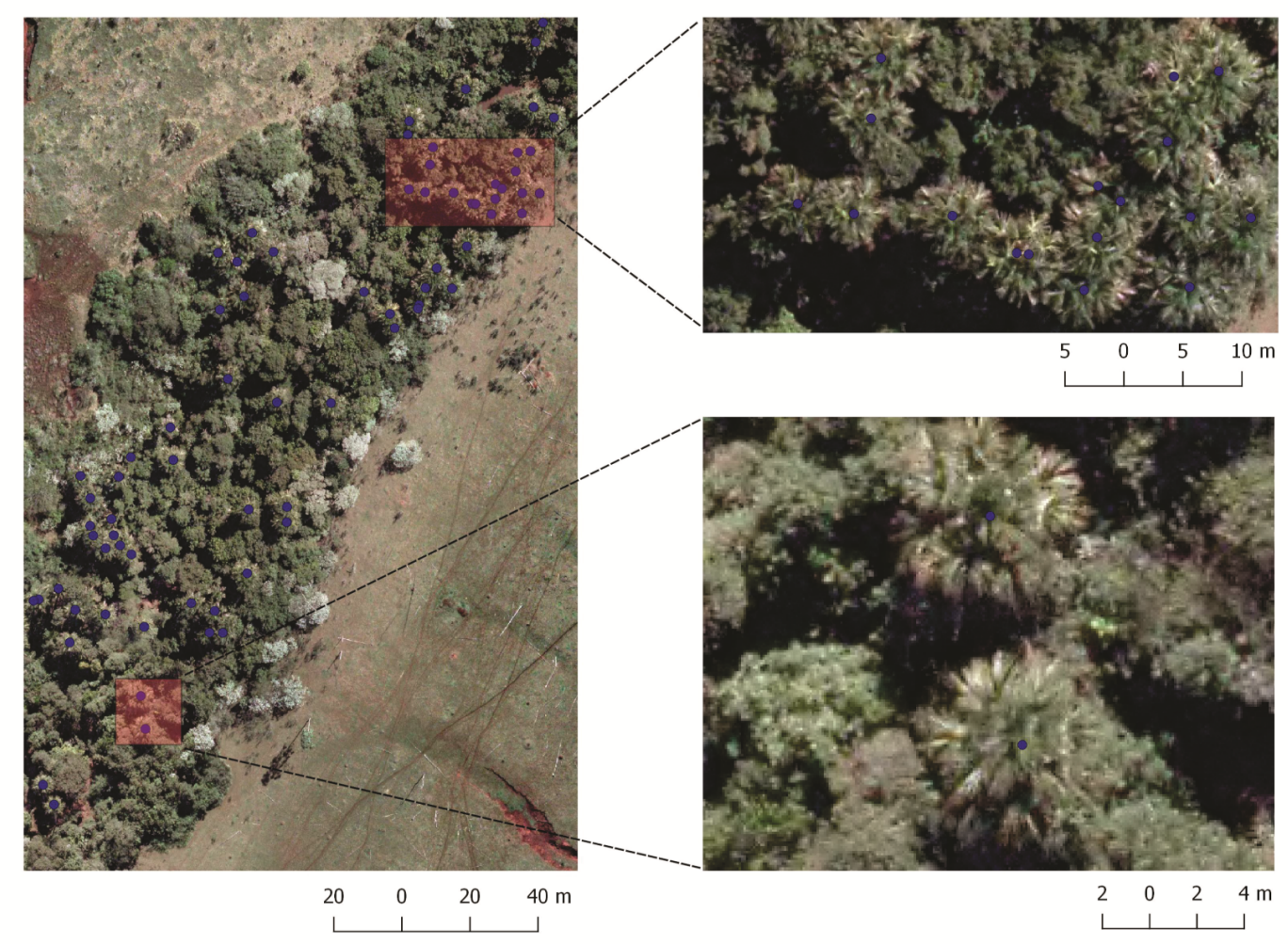

Fig. 3. Examples of the labeled dataset. M. flexuosa palm-trees are represented with blue dots. 


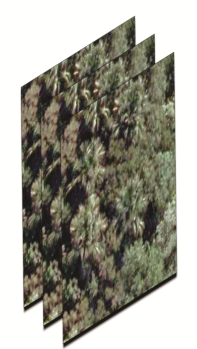

(a) Input image

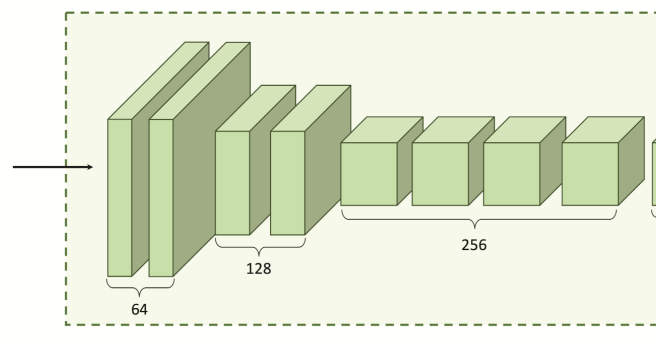

(b) Feature map extraction

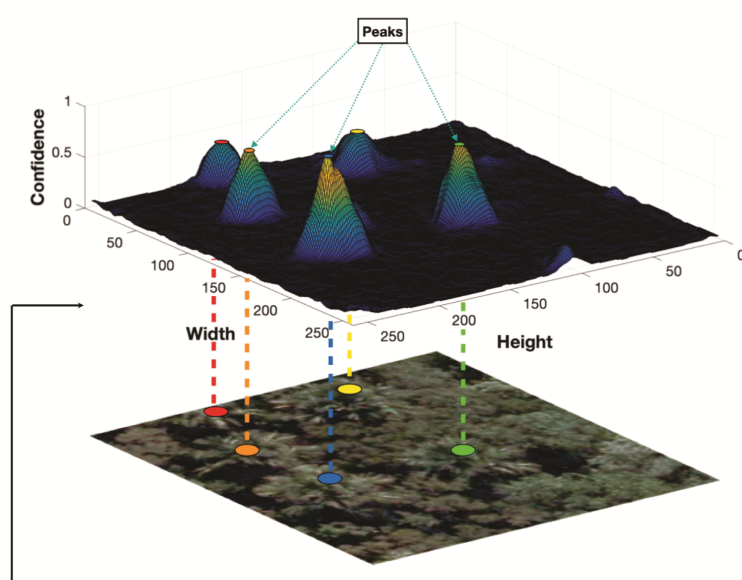

(e) Object detection

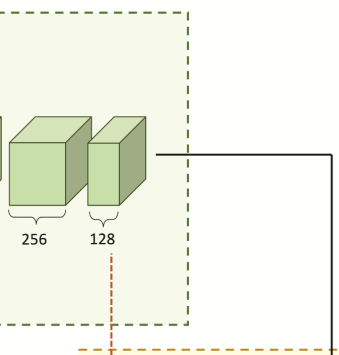

POOLING
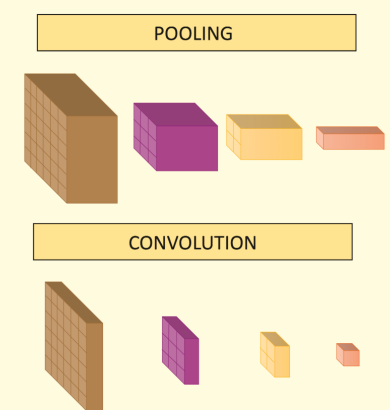

UPSAMPLING | CONCATENATION

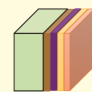

(c) Pyramid Pooling Module (PPM)

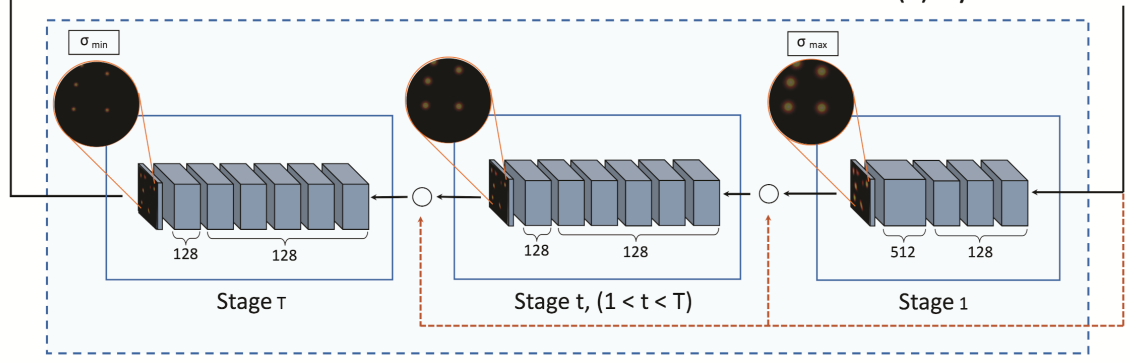

(d) Multi-Stage Module (MSM)

Fig. 4. Proposed CNN. The feature map (b) is extracted from the input image (a) and improved by the PPM (c). The result is used as input at the MSM step (d), where T stages enhance the prediction positions (e).

early phases of the experiment, different values for $t$ were adopted to improve its robustness. Finally, the tree location is estimated by the peaks of the confidence map (Figure 5). These peaks are considered the local maximum of the confidence map, representing a high probability of a tree occurrence. The $\mathrm{p}=(\mathrm{xp}, \mathrm{yp})$ is considered as a local maximum if $\mathrm{CT}(\mathrm{p})>\mathrm{CT}(\mathrm{v})$ for all neighbors $\mathrm{v}$. Here, $\mathrm{v}$ is given by (xp1, yp) or (xp, yp1).

A peak in the confidence map is defined as a real tree if $\mathrm{CT}(\mathrm{p})$ $>$ t. To prevent the network from confusing trees in a nearby range from each other, a distance of $t$ is defined. For this study, $\mathrm{t}$ equal to 1 pixel and $\mathrm{t}$ equal to 0.35 were defined as valid metrics. These values were defined during a previous experimental phase.

Experimental Setup. For the experimental setup, the RGB ortohomosaics were separated into training, validation, and testing, respectively (Figure 6). They were split into nonoverlapping patches of $256 \times 256$ pixels (25.6 m x 25.6 $\mathrm{m})$. The patches were then divided into training $(42.3 \%)$, validation $(34.5 \%)$, and testing $(23.2 \%)$ sets. Table 1 lists the number of samples (trees) and image patches, and Figure 6 displays examples of the orthomosaics used to extract the datasets. For the training process, the $\mathrm{CNN}$ was initialized with pre-trained weights from ImageNet and a Stochastic Gradient Descent optimizer was applied with a moment equal to 0.9. For this, the validation set was used to adjust the learning rate and the number of epochs, which were set to 0.001 and 100 , respectively.

Table 1. Description of the training, validation, and testing sets of the M. flexuosa palms-trees dataset.

\begin{tabular}{ccc}
\hline Dataset & Number of Patches & Number of Samples (Palm-Trees) \\
\hline Training & 590 & 1,784 \\
Validation & 481 & 2,296 \\
Testing & 323 & 1,254 \\
\hline
\end{tabular}




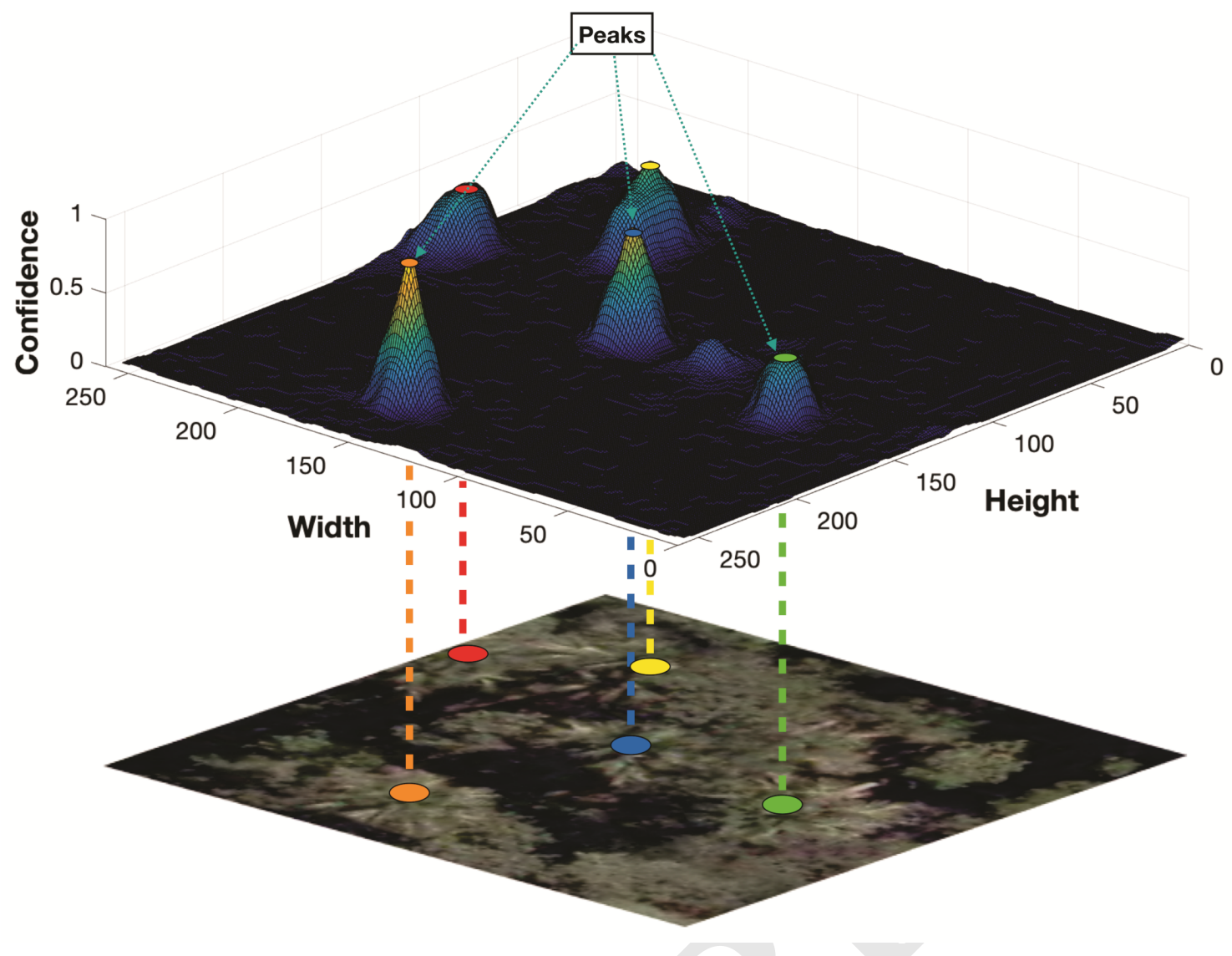

Fig. 5. Tree localization example from a refined confidence map.

The performance of the proposed network was assessed with the following metrics: mean absolute error (MAE); precision $(\mathrm{P})$; recall $(\mathrm{R})$, and; F1-measure (F1). The results were compared with Faster R-CNN and RetinaNet methods. Since these methods are based on bounding boxes, the plant position $(\mathrm{x}, \mathrm{y})$ from the labeled ground truth was used as a center of the box. The correct size of the box corresponds with the size occupied by the tree canopy. To perform this comparison, the same conjuncts of training, validation, and testing datasets were adopted for the three methods. Likely, an inverse process was applied during the test phase, where the position of the tree was obtained by the center of the point inside the predicted bounding-box of the RetinaNet and Faster R-CNN methods. This allowed applying the same metrics (MAE, P, R, and F1) for comparing the performances of each neural network.

\section{Results}

Validation of the Parameters. The proposed approach parameters $\sigma \min , \sigma \max$, and the number of stages $\mathrm{T}$, are responsible for refining the prediction map. Initially, the influence of these parameters was evaluated on the M. flexuosa palm trees validation set. Table 2 shows the evaluation of the number of stages $\mathrm{T}$ used in the MSM refinement phase. In this experiment, the values of $\sigma \min =1, \sigma \max =4$ and ranges $\mathrm{T}$ from 1 to 5 were set, and it was discovered that $\mathrm{T}=4$ achieved the best performance among the number of analyzed stages, reaching an MAE of 0.852 trees and an
F1-measure of $87.1 \%$.

Table 2. Influence of the number of stages $(T)$ in counting and detection of $M$. flexuosa palms-trees $(\sigma \min =1$ and $\sigma \max =4$ were adopted $)$.

\begin{tabular}{ccccc}
\hline Stages (T) & MAE & Precision (\%) & Recall (\%) & F1-measure (\%) \\
\hline 1 & 0.933 & 85.1 & 86.4 & 83.8 \\
2 & 0.943 & 93.5 & 83.6 & 86.9 \\
4 & 0.852 & 91.5 & 85.5 & 87.1 \\
5 & 0.966 & 93.9 & 83.1 & 86.6 \\
\hline
\end{tabular}

The values of $\sigma \min$ and $\sigma$ max applied in the refinement stage were also evaluated. For this, the number of stages $\mathrm{T}=4$ was adopted in the subsequent steps since it obtained the best results in the previous experiment (see Table 2). Since the $\sigma$ minvalues represent the dispersion of the density maps around the center of the trees, it was found that smaller values do not correctly cover the trees and, therefore, can impair the detection. On the other hand, higher $\sigma$ min values are also harmful as they cover more than one tree per area. Thus, the best results were obtained with $\sigma \max =4$, indicating that it fits better with the M. flexuosa palms-trees characteristics, and generates a more accurate refinement map.

Table 3 presents the evaluation of different values of $\sigma \mathrm{min}$ responsible for the last stage of the MSM. For this, $\sigma \max =$ 4 and $\mathrm{T}=4$ were adopted since they obtained better results in the previous experiments (Tables 2 and 3 ). When $\sigma \mathrm{min}=$ 1 , the proposed approach returned the best performance among the analyzed values. Therefore, the refinement step implemented with values of $\sigma \min =1, \sigma \max =4$, and $\mathrm{T}=4$ 


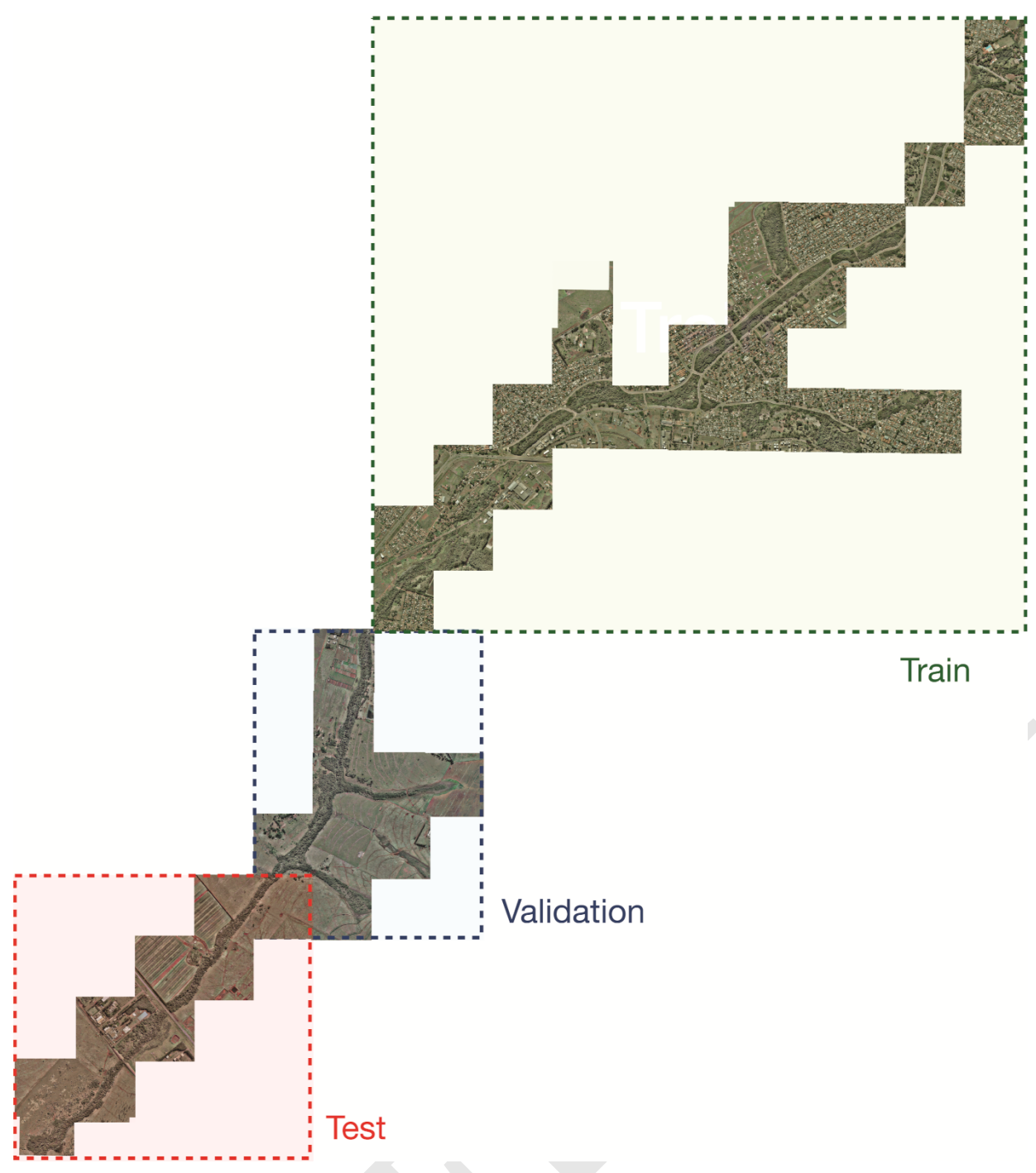

Fig. 6. Training, validation, and testing datasets separated per region.

generated a more accurate refinement to the validation set.

Table 3. Influence of the $\sigma$ max in counting and detection of M. flexuosa palms-trees $(\sigma \min =1$ and stages $\mathrm{T}=4$ were adopted $)$.

\begin{tabular}{ccccc}
\hline$\sigma \max$ & MAE & Precision (\%) & Recall (\%) & F1-measure (\%) \\
\hline 3 & 0.931 & 86.7 & 88.7 & 85.8 \\
4 & 0.852 & 91.5 & 85.5 & 87.1 \\
5 & 1.611 & 91.4 & 69.6 & 76.8 \\
\hline
\end{tabular}

Table 4. Influence of the $\sigma \min$ in counting and detection of M. flexuosa palms-trees ( $\sigma \max =4$ and stages $\mathrm{T}=4$ were used).

\begin{tabular}{ccccc}
\hline$\sigma \min$ & MAE & Precision $(\%)$ & Recall $(\%)$ & F1-measure $(\%)$ \\
\hline 0.75 & 1.879 & 90.7 & 64.9 & 73.3 \\
1 & 0.852 & 91.5 & 85.5 & 87.1 \\
1.5 & 1.012 & 83.9 & 88.9 & 84.3 \\
2 & 1.671 & 72.4 & 91 & 77.6 \\
\hline
\end{tabular}

\section{Comparative Results Between Object Detection Meth-}

ods. The proposed method returned better performance when compared with different object detection methods like Faster R-CNN and RetinaNet. The MAE, precision, recall, and F1-measure metrics were calculated for each of them, and the results are displayed in Table 5. The proposed approach achieved high precision and good F-measure values but returned a slight-lower recall value when confronted with them. Nonetheless, it is essential to consider the tradeoff in recall difference $(-6.58 \%$ from the Faster R-CNN and $12.35 \%$ from the RetinaNet) with the precision difference (+14.52 from the Faster R-CNN and $+35.49 \%$ from the RetinaNet).

Since the F1-measure uses both the precision and recall values to compute the test results, it can be assumed that the proposed approach performs better and returns a better balance between true-positive predicted and true-positive rates concerning the identification of palm-trees. Nonetheless, the results are consistent with recent literature where object detection applications were applied for the identification of single tree-species $(8,9,68,70)$; but performed in the non-RGB image domain. The low precision values for the bounding box method may be explained by a high density of objects (i.e., M. flexuosa palm-trees). This condition is described as problematic for deep networks based on 
these characteristics, especially when the boxes have high intersections with similar objects (71).

Table 5. Comparative results between our method and Faster R-CNN and RetinaNet.

\begin{tabular}{ccccc}
\hline Method & MAE & Precision (\%) & Recall (\%) & F1-measure (\%) \\
\hline Faster R-CNN & 0.984 & 79 & 90.8 & 81.7 \\
RetinaNet & 3.761 & 58 & 96.6 & 69.8 \\
Proposed Method & 0.758 & 93.5 & 84.2 & 86.9 \\
\hline
\end{tabular}

To verify the potential of the proposed approach in real-time processing, a comparison of its performance with other state-of-the-art methods was conducted. Table 6 shows the average processing time and standard deviation for 100 images of the test set. The values of $\sigma \min =1, \sigma \max =4$ and $\mathrm{T}=4$ were used to obtain the best performance in previous experiments. The results show that the approach can achieve real-time processing, delivering image detection in 0.073 seconds with a standard deviation of 0.002 using a GPU. Similarly, RetinaNet and Faster R-CNN methods obtained an average detection time and standard deviation of 0.057 , 0.046 , and $0.002,0.001$, respectively.

Figure 7 presents the qualitative results of the proposed method where the annotations of $\mathrm{M}$. flexuosa palm-trees are marked with yellow circles, and the blue dots indicate the correctly detected positions. This approach correctly detects the M. flexuosa palm trees in different capture conditions, such as overlapping trees (Figure 7(a)), partial occlusion of the treetops (Figure 7(b)), and highly dense vegetation areas (Figure 7(c)), highlighted by orange circles. Moreover, the predicted positions have a satisfactory level of accuracy, generating detection (blue dots) close to the annotations (center of the yellow circles).

Although the method obtained good results in the detection of M. flexuosa palm trees, it faces some challenges. Figure 8 presents areas where the incorrect detections are shown by the red circles. The main challenge is the detection of trees with a high level of occlusion at the image boundary or by overlapping of trees (highlighted by the orange circles). However, even in these few cases, the proposed approach can correctly detect most of the palm-trees.

The visual comparison of the palm-tree detection approaches is shown in Figure 9. Column (a) displays the detections obtained by the proposed method, while Columns (b) and (c) are related to the compared methods: Faster R-CNN and RetinaNet, respectively. The approach that obtained the worst result was RetinaNet (Figure 9(c)), generating many falsepositives (red dots) close to the M. flexuosa palm trees detections. On the other hand, Faster R-CNN (Figure 9(b)), despite having fewer false-positives, did not properly learn the characteristics of the palm-trees and incorrectly detected other tree species among them. Following the quantitative results shown in Table 5, the proposed approach has the greater precision in detecting M. flexuosa palm-trees (Figure 9(a)), while having the least number of incorrect detections (falsepositives).

\section{Discussion}

This study demonstrated a feasible method to automatically map single palm-tree species of the M. flexuosa plant genus using an RGB imagery dataset. Mauritia flexuosa frequently occurs at low elevations, with high density on river banks and lake margins, around water sources, and in inundated or humid areas (64). This is one of the most widely distributed palm trees in South America, Brazil. This species occurs in the Amazon region, Caatinga, Cerrado, and Pantanal, and is one of the palm trees mostly used by humans, being an important item in the diet of many indigenous groups and rural communities (64).

Mapping M. flexuosa palm-trees is an important practice for multiple regions of South America, like Brazil, where this plant is viewed as a valuable resource. This palm is widely used for several purposes, is considered a species of multiple use (62), occurs in areas of "Veredas", considered protected by the Brazilian forest code, but there is still a great lack of characterization of the habitats of this species in this country. Mapping and identifying populations of palm M.flexuosa is relevant because it is a reliable indicator of water resources, such as streams inside dense gallery forests, slow-flowing swamp surface water, and shallow groundwater in the Cerrado region, vital for humans and wildlife, besides being a valuable source of several non-timber forest products. The approach provides useful information for sustainable economic use and conservation.

As described, single tree species identification is a challenging task even for state-of-the-art deep neural networks when considering only RGB imagery. Mainly because forest environments are constituted by multiple spectral spatial information, overlapping canopies, leaves and branches, size, growth stages, and density, among others. In this manner, studies considered different data to help solve this issue like density point information, canopy height, digital terrain and surface models, spectral divergence, etc. $(4,15,32,40,54)$. Regardless, in this paper, it is proposed a simplification of this process by adopting little input information (i.e., label features such as points and RGB imagery) and a robust method that once trained, can rapidly perform and resolve the said task even in a real-time context.

The results of the present approach achieved satisfactory precision $(93.5 \%)$, recall $(84.2 \%)$, and F1-measure (86.9\%) values, respectively), and a small MAE (0.758 tree). Studies that applied deep neural networks for detecting other types of arboreal vegetation returned approximated metrics. For the identification of citrus-tree, a CNN method was able to provide $96.2 \%$ accuracy (16), and in oil palm-tree detection, a deep neural network implementation returned an accuracy of $96.0 \%$ (Li et al., 2019). One different kind of palm trees than the ones evaluated in our dataset was investigated with a modification of the AlexNet CNN architecture and returned high prediction values $\left(\mathrm{R}^{2}=0.99\right.$, with the relative error between $2.6 \%$ to $9.2 \%$ ) (70). A study (9) achieved an accuracy higher than $90 \%$ to detect single tree-species using the RetinaNet and RGB images. However, in the aforementioned papers, the tree density patterns are differently from ours, and the 


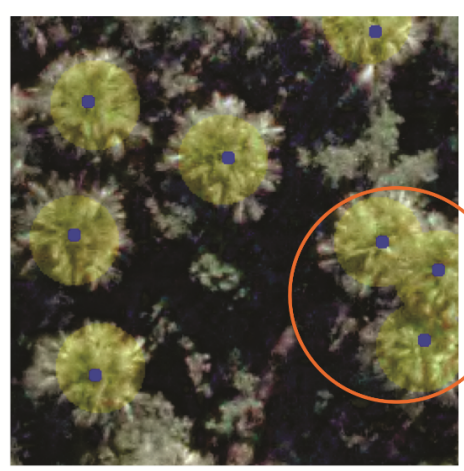

(a) Overlapping

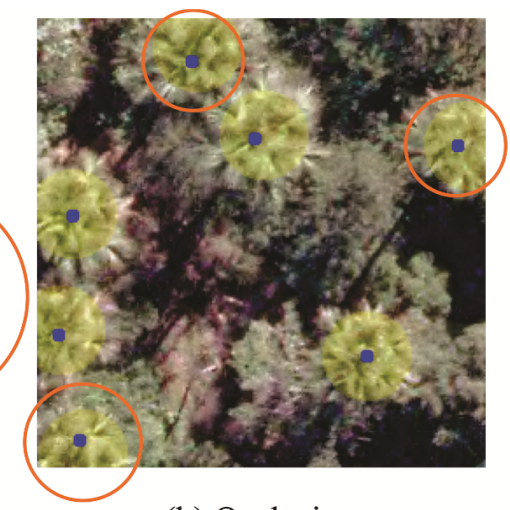

(b) Occlusion

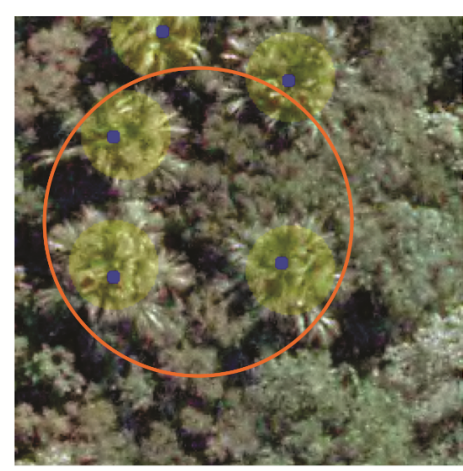

(c) High vegetation

Fig. 7. Qualitative results of the proposed method in three scenes: (a) an example of the detected nearby trees with overlapping, (b) detected trees with parts of the canopy occluded at the edge of the image, and (c) demons detected trees in areas of high vegetation. The orange circles highlight the detections.
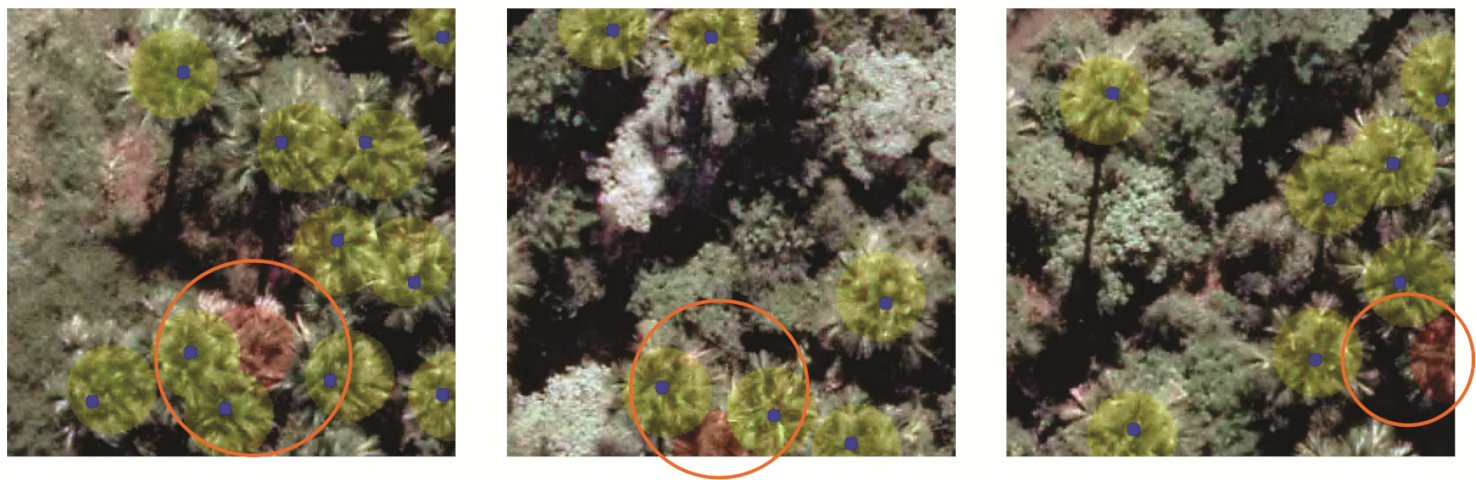

Fig. 8. Examples of the challenges faced by our method in the M. flexuosa palm-tree detection task. The orange circles indicate challenging detections.
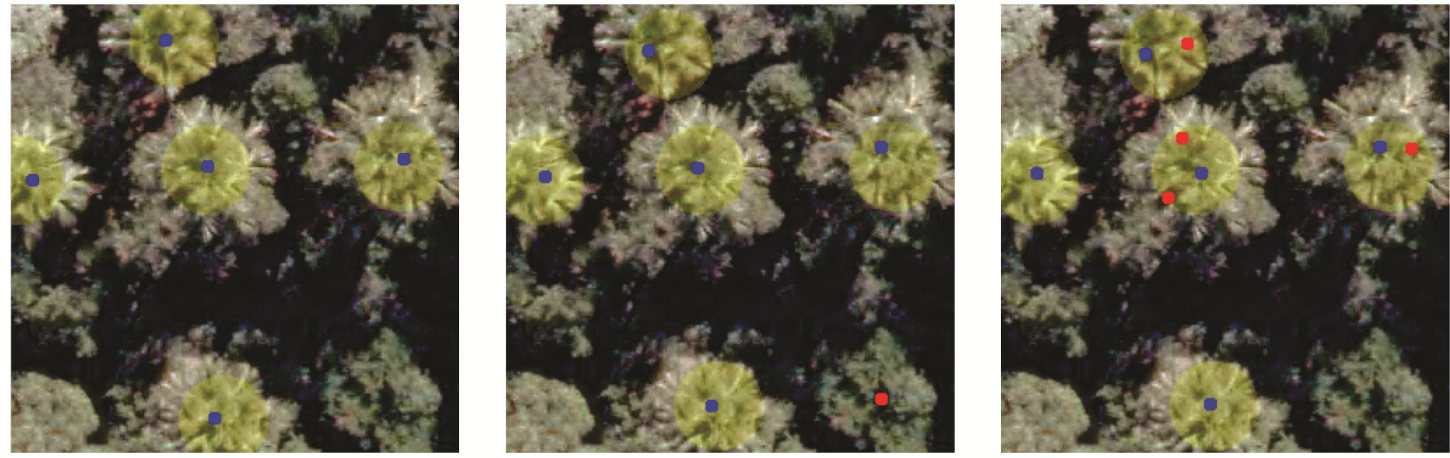

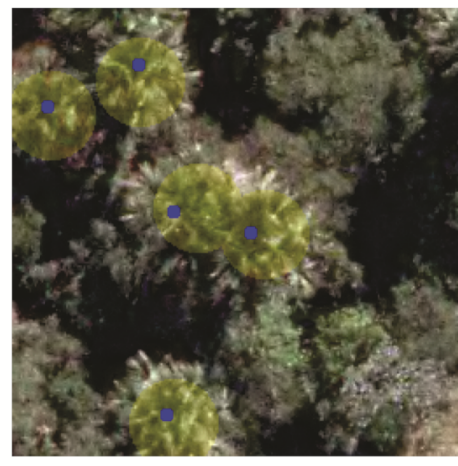

(a) Proposed approach

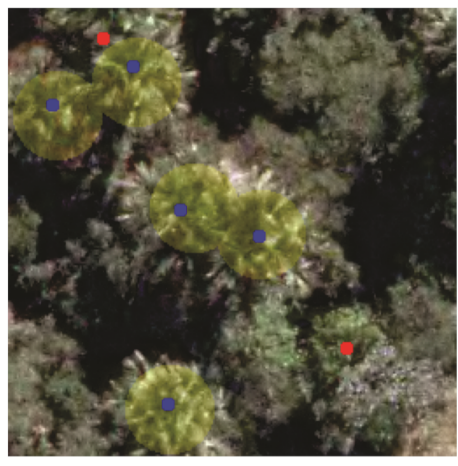

(b) Faster R-CNN

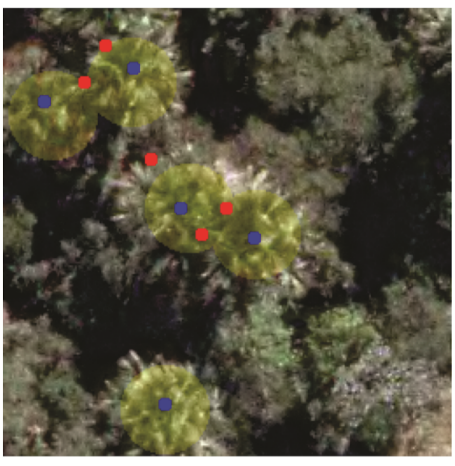

(c) RetinaNet

Fig. 9. Visual comparison of the analyzed methods. (a) shows the detections obtained by the proposed approach; (b) indicates the detections of the Faster R-CNN and; (c) demonstrates the detections of the RetinaNet. Blue and red points correspond to correct and incorrect detection positions, respectively, and the yellow circle to M. flexuosa palms-trees annotation. 
Table 6. Processing time evaluation of the analyzed approaches.

\begin{tabular}{ccccc}
\hline Method & CPU & & GPU & \\
& Average Time (sec) & Standard deviation & Average Time (sec) & Standard deviation \\
\hline Faster R-CNN & 1.57 & 0.031 & 0.05 & 0.001 \\
RetinaNet & 1.93 & 0.028 & 0.06 & 0.002 \\
Proposed Method & 1.26 & 0.051 & 0.07 & 0.002 \\
\hline
\end{tabular}

evaluated individual trees are more spaced from each other, which makes a simpler object detection problem.

In the described manner, the proposed method may help in mapping the $\mathrm{M}$. flexuosa palm-tree with little computational load and high accuracy. Since this approach can compute point features as labeled objects, it reduces the amount of label-work required from the human counterpart. Additionally, the method provided a fast solution to detect the palm tree's location with a delivering image detection of $0.073 \mathrm{sec}-$ onds and a standard deviation of 0.002 using a GPU. This information is essential for properly calculating the cost and effectiveness of the method. The presented approach may help new research while providing primary information for exploring environmental management practices in the experiment context (i.e., evaluating a keystone tree species). The proposed method could also be incorporated into areas and regions to help detect the $M$. flexuosa palm tree and contribute to decision-making conservation measures of the said species.

\section{Conclusions}

This paper presents an approach based on deep networks to map single species of fruit palm trees (Mauritia flexuosa) in aerial RGB imagery. According to the performance assessment, the method returned an MAE of 0.75 trees and F1measure of $86.9 \%$. A comparative study also shows that the proposed method returned better accuracy than state-ofthe-art methods like Faster R-CNN and RetinaNet under the same experimental conditions. Besides, this approach took a shorter time to detect the palm-trees with 0.073 seconds for delivering image detection and achieved a standard deviation of 0.002 using the GPU. In future implementations, it should be possible to add new strategies in this CNN architecture to overcome challenges regarding other tree patterns. Still, the identification of individual species can help to assist in both monitoring and mapping important singular species. As such, the proposed method may assist in new research for the forest remote sensing community that includes data obtained with RGB sensors.

\section{ACKNOWLEDGEMENTS}

The authors acknowledge the support of the UFMS (Federal University of Mato Grosso do Sul) and Coordenação de Aperfeiçoamento de Pessoal de Nível Superior (CAPES) (Finance Code 001)

\section{FUNDING}

This research was partially funded by CNPq (p: 433783/2018-4, 303559/2019-5, 304052/2019-1, and 310517/2020-6) and CAPES Print (p: 88881.311850/201801). The authors acknowledge the support of the UFMS (Federal Universityof Mato Grosso do Sul) and CAPES (Finance Code 001), and NVIDIAC for the donation of the Titan $\mathrm{X}$ graphics card used in the experiments.

\section{CONFLICTS OF INTEREST}

The authors declare no conflict of interest. The funders had no role in the design of the study; in the collection, analyses, or interpretation of data; in the writing of the manuscript, or in the decision to publish the results.

\section{Bibliography}

1. Roope Näsi, Eija Honkavaara, Päivi Lyytikäinen-Saarenmaa, Minna Blomqvist, Paula Litkey, Teemu Hakala, Niko Viljanen, Tuula Kantola, Topi Tanhuanpää, and Markus Holopainen. Using UAV-based photogrammetry and hyperspectral imaging for mapping bark beetle damage at tree-level. Remote Sensing, 7(11):15467-15493, nov 2015. doi: 10.3390/rs71115467.

2. Alejandro Navarro, Mary Young, Blake Allan, Paul Carnell, Peter Macreadie, and Daniel lerodiaconou. The application of unmanned aerial vehicles (UAVs) to estimate aboveground biomass of mangrove ecosystems. Remote Sensing of Environment, 242:111747, jun 2020. doi: 10.1016/j.rse.2020.111747.

3. Bruna Paolinelli Reis, Sebastião Venâncio Martins, Elpídio Inácio Fernandes Filho, Tathiane Santi Sarcinelli, José Marinaldo Gleriani, Gustavo Eduardo Marcatti, Helio Garcia Leite, and Melinda Halassy. Management recommendation generation for areas under forest restoration process through images obtained by UAV and LiDAR. Remote Sensing, 11 (13):1508, June 2019. doi: 10.3390/rs11131508.

4. Ninni Saarinen, Mikko Vastaranta, Roope Näsi, Tomi Rosnell, Teemu Hakala, Eija Honkavaara, Michael Wulder, Ville Luoma, Antonio Tommaselli, Nilton Imai, Eduardo Ribeiro, Raul Guimarães, Markus Holopainen, and Juha Hyyppä. Assessing biodiversity in boreal forests with UAV-based photogrammetric point clouds and hyperspectral imaging. Remote Sensing, 10(2):338, February 2018. doi: 10.3390/rs10020338.

5. Quanlong Feng, Jiantao Liu, and Jianhua Gong. UAV remote sensing for urban vegetation mapping using random forest and texture analysis. Remote Sensing, 7(1):1074-1094, jan 2015. doi: 10.3390/rs70101074.

6. Konstantinos P. Ferentinos. Deep learning models for plant disease detection and diagnosis. Computers and Electronics in Agriculture, 145:311-318, feb 2018. doi: 10.1016/j.compag. 2018.01.009.

7. Ximena Tagle Casapia, Lourdes Falen, Harm Bartholomeus, Rodolfo Cárdenas, Gerardo Flores, Martin Herold, Eurídice N. Honorio Coronado, and Timothy R. Baker. Identifying and quantifying the abundance of economically important palms in tropical moist forest using UAV imagery. Remote Sensing, 12(1):9, dec 2019. doi: 10.3390/rs12010009.

8. Linyuan Li, Jun Chen, Xihan Mu, Weihua Li, Guangjian Yan, Donghui Xie, and Wuming Zhang. Quantifying understory and overstory vegetation cover using UAV-based RGB imagery in forest plantation. Remote Sensing, 12(2):298, jan 2020. doi: 10.3390/rs12020298.

9. Anderson Aparecido dos Santos, José Marcato Junior, Márcio Santos Araújo, David Robledo Di Martini, Everton Castelão Tetila, Henrique Lopes Siqueira, Camila Aoki, Anette Eltner, Edson Takashi Matsubara, Hemerson Pistori, Raul Queiroz Feitosa, Veraldo Liesenberg, and Wesley Nunes Gonçalves. Assessment of CNN-based methods for individual tree detection on images captured by RGB cameras attached to UAVs. Sensors, 19(16):3595, August 2019. doi: 10.3390/s19163595.

10. Gabriela Takahashi Miyoshi, Nilton Nobuhiro Imai, Antonio Maria Garcia Tommaselli, Marcus Vinícius Antunes de Moraes, and Eija Honkavaara. Evaluation of hyperspectral multitemporal information to improve tree species identification in the highly diverse atlantic forest. Remote Sensing, 12(2):244, jan 2020. doi: 10.3390/rs12020244.

11. Giorgio Morales, Guillermo Kemper, Grace Sevillano, Daniel Arteaga, Ivan Ortega, and Joel Telles. Automatic segmentation of mauritia flexuosa in unmanned aerial vehicle (UAV) imagery using deep learning. Forests, 9(12):736, nov 2018. doi: 10.3390/f9120736.

12. Matthew Voss and Ramanathan Sugumaran. Seasonal effect on tree species classification in an urban environment using hyperspectral data, LiDAR, and an object- oriented approach. Sensors, 8(5):3020-3036, May 2008. doi: 10.3390/s8053020.

13. Hans-Erik Andersen, Stephen E Reutebuch, and Robert J McGaughey. A rigorous assessment of tree height measurements obtained using airborne lidar and conventional field methods. Canadian Journal of Remote Sensing, 32(5):355-366, 2006. doi: 10.5589/ m06-030.

14. Selina Ganz, Yannek Käber, and Petra Adler. Measuring tree height with remote sensing-a comparison of photogrammetric and LiDAR data with different field measurements. Forests, 10(8):694, aug 2019. doi: 10.3390/f10080694.

15. A. Ozdarici-Ok. Automatic detection and delineation of citrus trees from VHR satellite imagery. International Journal of Remote Sensing, 36(17):4275-4296, aug 2015. doi: 10.1080/01431161.2015.1079663.

16. Ovidiu Csillik, John Cherbini, Robert Johnson, Andy Lyons, and Maggi Kelly. Identification of citrus trees from unmanned aerial vehicle imagery using convolutional neural networks. Drones, 2(4):39, nov 2018. doi: 10.3390/drones2040039.

17. Adilson Berveglieri, Nilton N. Imai, Antonio M.G. Tommaselli, Baltazar Casagrande, and Eija Honkavaara. Successional stages and their evolution in tropical forests using multi-temporal photogrammetric surface models and superpixels. ISPRS Journal of Photogrammetry and Remote Sensing, 146:548-558, dec 2018. doi: 10.1016/j.isprsjprs.2018.11.002.

18. Jingjing Cao, Wanchun Leng, Kai Liu, Lin Liu, Zhi He, and Yuanhui Zhu. Object-based mangrove species classification using unmanned aerial vehicle hyperspectral images and digital surface models. Remote Sensing, 10(2):89, jan 2018. doi: 10.3390/rs10010089.

19. Ben G. Weinstein, Sergio Marconi, Stephanie Bohlman, Alina Zare, and Ethan White. Individual tree-crown detection in RGB imagery using semi-supervised deep learning neural networks. Remote Sensing, 11(11):1309, June 2019. doi: 10.3390/rs11111309. 
20. Daliana Lobo Torres, Raul Queiroz Feitosa, Patrick Nigri Happ, Laura Elena Cué La Rosa, José Marcato Junior, José Martins, Patrik Olã Bressan, Wesley Nunes Gonçalves, and Veraldo Liesenberg. Applying fully convolutional architectures for semantic segmentation of a single tree species in urban environment on high resolution UAV optical imagery. Sensors, 20(2):563, jan 2020. doi: 10.3390/s20020563.

21. M CLARK, D ROBERTS, and D CLARK. Hyperspectral discrimination of tropical rain forest tree species at leaf to crown scales. Remote Sensing of Environment, 96(3-4):375-398, jun 2005. doi: 10.1016/j.rse.2005.03.009.

22. Trevor G. Jones, Nicholas C. Coops, and Tara Sharma. Assessing the utility of airborne hyperspectral and LiDAR data for species distribution mapping in the coastal pacific northwest, canada. Remote Sensing of Environment, 114(12):2841-2852, dec 2010. doi: 10.1016/j.rse.2010.07.002

23. Matthew Colgan, Claire Baldeck, Jean-Baptiste Féret, and Gregory Asner. Mapping savanna tree species at ecosystem scales using support vector machine classification and BRDF correction on airborne hyperspectral and LiDAR data. Remote Sensing, 4(11):34623480, nov 2012. doi: 10.3390/rs4113462.

24. Liangyun Liu, Bowen Song, Su Zhang, and Xinjie Liu. A novel principal component analysis method for the reconstruction of leaf reflectance spectra and retrieval of leaf biochemical contents. Remote Sensing, 9(11):1113, oct 2017. doi: 10.3390/rs9111113.

25. Julia Maschler, Clement Atzberger, and Markus Immitzer. Individual tree crown segmentation and classification of 13 tree species using airborne hyperspectral data. Remote Sensing, 10(8):1218, aug 2018. doi: 10.3390/rs10081218.

26. Andrew Hennessy, Kenneth Clarke, and Megan Lewis. Hyperspectral classification of plants: A review of waveband selection generalisability. Remote Sensing, 12(1):113, jan 2020. doi: $10.3390 / \mathrm{rs} 12010113$

27. Michele Dalponte, Hans Ole Ørka, Liviu Theodor Ene, Terje Gobakken, and Erik Næsset Tree crown delineation and tree species classification in boreal forests using hyperspectral and ALS data. Remote Sensing of Environment, 140:306-317, jan 2014. doi: 10.1016/j.rse. 2013.09.006.

28. Michele Dalponte, Lorenzo Bruzzone, and Damiano Gianelle. Tree species classification in the southern alps based on the fusion of very high geometrical resolution multispectral/hyperspectral images and LiDAR data. Remote Sensing of Environment, 123:258-270, aug 2012. doi: 10.1016/j.rse.2012.03.013.

29. Moses Azong Cho, Renaud Mathieu, Gregory P. Asner, Laven Naidoo, Jan van Aardt, Abe Ramoelo, Pravesh Debba, Konrad Wessels, Russell Main, Izak P.J. Smit, and Barend Erasmus. Mapping tree species composition in south african savannas using an integrated airborne spectral and LiDAR system. Remote Sensing of Environment, 125:214-226, oct 2012. doi: 10.1016/j.rse.2012.07.010.

30. Bogdan Apostol, Marius Petrila, Adrian Lorenţ, Albert Ciceu, Vladimir Gancz, and Ovidiu Badea. Species discrimination and individual tree detection for predicting main dendrometric characteristics in mixed temperate forests by use of airborne laser scanning and ultra-high-resolution imagery. Science of The Total Environment, 698:134074, jan 2020. doi: 10.1016/j.scitotenv.2019.134074.

31. Markus Immitzer, Clement Atzberger, and Tatjana Koukal. Tree species classification with random forest using very high spatial resolution 8-band WorldView-2 satellite data. Remote Sensing, 4(9):2661-2693, sep 2012. doi: 10.3390/rs4092661.

32. Steven E. Franklin and Oumer S. Ahmed. Deciduous tree species classification using object-based analysis and machine learning with unmanned aerial vehicle multispectra data. International Journal of Remote Sensing, 39(15-16):5236-5245, aug 2017. doi: 10.1080/01431161.2017.1363442.

33. Michele Dalponte, Hans Ole Orka, Terje Gobakken, Damiano Gianelle, and Erik Naesset. Tree species classification in boreal forests with hyperspectral data. IEEE Transactions on Geoscience and Remote Sensing, 51(5):2632-2645, may 2013. doi: 10.1109/tgrs.2012. 2216272.

34. Nathalie Guimarães, Luís Pádua, Pedro Marques, Nuno Silva, Emanuel Peres, and Joaquim J. Sousa. Forestry remote sensing from unmanned aerial vehicles: A review focusing on the data, processing and potentialities. Remote Sensing, 12(6):1046, mar 2020. doi: $10.3390 / \mathrm{rs} 12061046$.

35. Mariana Belgiu and Lucian Drăguţ. Random forest in remote sensing: A review of applications and future directions. ISPRS Journal of Photogrammetry and Remote Sensing, 114 24-31, apr 2016. doi: 10.1016/j.isprsjprs.2016.01.011.

36. Roope Näsi, Eija Honkavaara, Minna Blomqvist, Päivi Lyytikäinen-Saarenmaa, Teemu Hakala, Niko Viljanen, Tuula Kantola, and Markus Holopainen. Remote sensing of bark beetle damage in urban forests at individual tree level using a novel hyperspectral camera from UAV and aircraft. Urban Forestry \& Urban Greening, 30:72-83, mar 2018. doi 10.1016/j.ufug.2018.01.010.

37. Somayeh Nezami, Ehsan Khoramshahi, Olli Nevalainen, Ilkka Pölönen, and Eija Honkavaara. Tree species classification of drone hyperspectral and RGB imagery with deep learning convolutional neural networks. Remote Sensing, 12(7):1070, mar 2020. doi 10.3390/rs12071070.

38. Olli Nevalainen, Eija Honkavaara, Sakari Tuominen, Niko Viljanen, Teemu Hakala, Xiaowei Yu, Juha Hyyppä, Heikki Saari, Ilkka Pölönen, Nilton Imai, and Antonio Tommaselli. Individual tree detection and classification with UAV-based photogrammetric point clouds and hyperspectral imaging. Remote Sensing, 9(3):185, feb 2017. doi: 10.3390/rs9030185.

39. Edwin Raczko and Bogdan Zagajewski. Comparison of support vector machine, random forest and neural network classifiers for tree species classification on airborne hyperspectral APEX images. European Journal of Remote Sensing, 50(1):144-154, January 2017. doi: 10.1080/22797254.2017.1299557.

40. Sakari Tuominen, Roope Näsi, Eija Honkavaara, Andras Balazs, Teemu Hakala, Niko Viljanen, Ilkka Pölönen, Heikki Saari, and Harri Ojanen. Assessment of classifiers and remote sensing features of hyperspectral imagery and stereo-photogrammetric point clouds for recognition of tree species in a forest area of high species diversity. Remote Sensing, 10(5):714, May 2018. doi: 10.3390/rs10050714.

41. Zhuli Xie, Yaoliang Chen, Dengsheng Lu, Guiying Li, and Erxue Chen. Classification of land cover, forest, and tree species classes with ZiYuan-3 multispectral and stereo data. Remote Sensing, 11(2):164, January 2019. doi: 10.3390/rs11020164.
42. Aaron E. Maxwell, Timothy A. Warner, and Fang Fang. Implementation of machine-learning classification in remote sensing: an applied review. International Journal of Remote Sensing, 39(9):2784-2817, feb 2018. doi: 10.1080/01431161.2018.1433343.

43. Lucas Prado Osco, Ana Paula Marques Ramos, Danilo Roberto Pereira, Érika Akemi Saito Moriya, Nilton Nobuhiro Imai, Edson Takashi Matsubara, Nayara Estrabis, Maurício de Souza, José Marcato Junior, Wesley Nunes Gonçalves, Jonathan Li, Veraldo Liesenberg, and José Eduardo Creste. Predicting canopy nitrogen content in citrus-trees using random forest algorithm associated to spectral vegetation indices from UAV-imagery. Remote Sensing, 11(24):2925, dec 2019. doi: 10.3390/rs11242925.

44. Julia Marrs and Wenge Ni-Meister. Machine learning techniques for tree species classification using co-registered LiDAR and hyperspectral data. Remote Sensing, 11(7):819, apr 2019. doi: $10.3390 / \mathrm{rs} 11070819$

45. Mohammad Imangholiloo, Ninni Saarinen, Lauri Markelin, Tomi Rosnell, Roope Näsi, Teemu Hakala, Eija Honkavaara, Markus Holopainen, Juha Hyyppä, and Mikko Vastaranta. Characterizing seedling stands using leaf-off and leaf-on photogrammetric point clouds and hyperspectral imagery acquired from unmanned aerial vehicle. Forests, 10(5):415, may 2019. doi: 10.3390/f10050415.

46. Tien Pham, Naoto Yokoya, Dieu Bui, Kunihiko Yoshino, and Daniel Friess. Remote sensing approaches for monitoring mangrove species, structure, and biomass: Opportunities and challenges. Remote Sensing, 11(3):230, January 2019. doi: 10.3390/rs1 1030230.

47. Weijia Li, Haohuan Fu, Le Yu, and Arthur Cracknell. Deep learning based oil palm tree detection and counting for high-resolution remote sensing images. Remote Sensing, 9(1): 22, dec 2016. doi: 10.3390/rs9010022.

48. Lei Ma, Yu Liu, Xueliang Zhang, Yuanxin Ye, Gaofei Yin, and Brian Alan Johnson. Deep learning in remote sensing applications: A meta-analysis and review. ISPRS Journal of Photogrammetry and Remote Sensing, 152:166-177, jun 2019. doi: 10.1016/j.isprsjprs. 2019.04.015.

49. Anastasiia Safonova, Siham Tabik, Domingo Alcaraz-Segura, Alexey Rubtsov, Yuriy Maglinets, and Francisco Herrera. Detection of fir trees (abies sibirica) damaged by the bark beetle in unmanned aerial vehicle images with deep learning. Remote Sensing, 11(6): 643, March 2019. doi: 10.3390/rs11060643.

50. Jürgen Schmidhuber. Deep learning in neural networks: An overview. Neural Networks, 61: 85-117, 2015. ISSN 0893-6080. doi: https://doi.org/10.1016/j.neunet.2014.09.003.

51. Andreas Kamilaris and Francesc X. Prenafeta-Boldú. Deep learning in agriculture: A survey. Computers and Electronics in Agriculture, 147:70-90, apr 2018. doi: 10.1016/j.compag. 2018.02.016.

52. Giorgio Morales, Guillermo Kemper, Grace Sevillano, Daniel Arteaga, Ivan Ortega, and Joel Telles. Automatic segmentation of mauritia flexuosa in unmanned aerial vehicle (UAV) imagery using deep learning. Forests, 9(12):736, November 2018. doi: 10.3390/f9120736.

53. Aditya Khamparia and Karan Mehtab Singh. A systematic review on deep learning architectures and applications. Expert Systems, 36(3):e12400, mar 2019. doi: 10.1111/exsy.12400.

54. C. Sothe, C. M. De Almeida, M. B. Schimalski, L. E. C. La Rosa, J. D. B. Castro, R. Q. Feitosa, M. Dalponte, C. L. Lima, V. Liesenberg, G. T. Miyoshi, and A. M. G. Tommaselli. Comparative performance of convolutional neural network, weighted and conventional support vector machine and random forest for classifying tree species using hyperspectral and photogrammetric data. GIScience \& Remote Sensing, 57(3):369-394, January 2020. doi: 10.1080/15481603.2020.1712102.

55. Joseph Redmon and Ali Farhadi. Yolov3: An incremental improvement, 2018.

56. Tsung-Yi Lin, Priya Goyal, Ross Girshick, Kaiming He, and Piotr Dollár. Focal loss for dense object detection, 2018.

57. Shaoqing Ren, Kaiming He, Ross Girshick, and Jian Sun. Faster r-cnn: Towards real-time object detection with region proposal networks, 2016

58. Karen Simonyan and Andrew Zisserman. Very deep convolutional networks for large-scale image recognition, 2015

59. Jean-Daniel Sylvain, Guillaume Drolet, and Nicolas Brown. Mapping dead forest cover using a deep convolutional neural network and digital aerial photography. ISPRS Journal o Photogrammetry and Remote Sensing, 156:14-26, October 2019. doi: 10.1016/j.isprsjprs. 2019.07.010.

60. Sean Hartling, Vasit Sagan, Paheding Sidike, Maitiniyazi Maimaitijiang, and Joshua Carron Urban tree species classification using a WorldView-2/3 and LiDAR data fusion approach and deep learning. Sensors, 19(6):1284, mar 2019. doi: 10.3390/s19061284.

61. Michael P Gilmore, Bryan A Endress, and Christa M Horn. The socio-cultural importance of mauritia flexuosa palm swamps (aguajales) and implications for multi-use management in two maijuna communities of the peruvian amazon. Journal of Ethnobiology and Ethnomedicine, 9(1):29, 2013. doi: 10.1186/1746-4269-9-29.

62. Ieda Maria Bortolotto, Geraldo Alves Damasceno-Junior, and Arnildo Pott. Preliminary list of native food plants from mato grosso do sul, brazil. Iheringia, Série Botânica, 73(Suppl): 101-116, March 2018. doi: 10.21826/2446-8231201873s101.

63. Yntze van der Hoek, Sara Álvarez Solas, and María Cristina Peñuela. The palm mauritia flexuosa, a keystone plant resource on multiple fronts. Biodiversity and Conservation, 28 (3):539-551, mar 2019. doi: 10.1007/s10531-018-01686-4.

64. Tânia da Silveira Agostini-Costa, Juliana Pereira Faria, Ronaldo Veloso Naves, and Roberto Fontes Vieira. Espécies Nativas da Flora Brasileira de Valor Econômico Atual ou Potencial Plantas para o Futuro - Região Centro-Oeste. Ministério do Meio Ambiente MMA, 2016. ISBN 9788577383092

65. Cesar Delgado, Guy Couturier, and Kember Mejia. Mauritia flexuosa(arecaceae: Calamoideae), an amazonian palm with cultivation purposes in peru. Fruits, 62(3):157-169, May 2007. doi: 10.1051/fruits:2007011.

66. Roxaneh Khorsand Rosa and Suzanne Koptur. New findings on the pollination biology of mauritia flexuosa (arecaceae) in roraima, brazil: Linking dioecy, wind, and habitat. American Journal of Botany, 100(3):613-621, March 2013. doi: 10.3732/ajb.1200446.

67. Jennifer A. Holm, Christopher J. Miller, and Wendell P. Cropper. Population dynamics of the dioecious amazonian PalmMauritia flexuosa: Simulation analysis of sustainable harvesting. Biotropica, 40(5):550-558, sep 2008. doi: 10.1111/j.1744-7429.2008.00412.x.

68. Lucas Prado Osco, Mauro dos Santos de Arruda, José Marcato Junior, Neemias Buceli da Silva, Ana Paula Marques Ramos, Érika Akemi Saito Moryia, Nilton Nobuhiro Imai, 
Danillo Roberto Pereira, José Eduardo Creste, Edson Takashi Matsubara, Jonathan Li, and Wesley Nunes Gonçalves. A convolutional neural network approach for counting and geolocating citrus-trees in UAV multispectral imagery. ISPRS Journal of Photogrammetry and Remote Sensing, 160:97-106, feb 2020. doi: 10.1016/j.isprsjprs.2019.12.010.

69. Hengshuang Zhao, Jianping Shi, Xiaojuan Qi, Xiaogang Wang, and Jiaya Jia. Pyramid scene parsing network, 2017.

70. K. Djerriri, M. Ghabi, M. S. Karoui, and R. Adjoudj. Palm trees counting in remote sensing imagery using regression convolutional neural network. In IGARSS 2018 - 2018 IEEE International Geoscience and Remote Sensing Symposium, volume 1, pages 2627-2630, 2018. doi: 10.1109/IGARSS.2018.8519188.

71. Eran Goldman, Roei Herzig, Aviv Eisenschtat, Oria Ratzon, Itsik Levi, Jacob Goldberger, and Tal Hassner. Precise detection in densely packed scenes, 2019. 